\title{
Hardness of nitric acid treated polyethylene followed by recrystallization
}

\author{
M. E. Cagiao, D. R. Rueda, and F. J. Baltá Calleja \\ Instituto de Estructura de la Materia, CSIC, Madrid, Spain
}

\begin{abstract}
The hardness variation of melt crystallized polyethylene as a consequence of controlled fuming nitric exposure has been investigated using the microindentation technique. This study complements previous results obtained using other reagents $\left(\mathrm{H}_{2} \mathrm{SO}_{4}\right.$, $\mathrm{ClHSO}_{3}$ ). After $\mathrm{HNO}_{3}$ exposure the microhardness of polyethylene decreases very rapidly, instead of increasing after the first hours of treatment. The hardness decrease is correlated to the volume fraction of interlamellar microvoids arising through selective acid digestion. For longer treatment times $(t>40 \mathrm{~h})$ the fragility of the material increases and the sample collapses under the indenter. The hardening of the degraded material after recrystallization from the melt is followed as a function of treatment time. The results are discussed in the light of the molecular mechanisms involved. Comparison of the experimental data with hardness calculations for ideal PE lamellar structures and chain extended dicarboxylic crystals implies that the major contribution to hardening is due to electron dense groups attachment at the surface of a mixed lamellar structure.
\end{abstract}

Key words: Microhardness, polyethylene, fuming nitric acid, dicarboxylic acids.

\section{Introduction}

Microindentation techniques, long used for routine hardness testing have been finding, in recent years, increasing application in the study of morphological changes within semicrystalline polymers $[1,2]$. Specifically, we have shown that the surface hardening of polyethylene through selective acid exposure (chlorosulfonic-, sulfuric acid) can be conveniently followed by the microindentation hardness technique $[3,4]$. The rate of hardness increase has been related to a preferential hardening of the crystalline lamellae due to crystal surface fixation of electron dense groups (sulphonic, chlorosulphonic). These groups contribute to cohesion-energy enhancement of lamellae, impede slippage of crystals and reduce the creep of the material during the test. The final hardening induced has been shown to be a function of thickness of the amorphous layer and of the temperature of treatment.

The purpose of the present communication is to supplement the above studies and report on the hardness investigation of polyethylene exposed to fuming nitric acid. The changes in microstructure and the detailed mechanism of fuming nitric attack to bulk polyethylene has been extensively discussed in the past [5-11]. The major issues may be summarized as follows: fuming nitric acid exposure, first, removes the disordered domains selectively inducing a weight-loss of material (10-70\%) depending on crystallinity. Second, it leaves the less reactive crystalline component as a residue containing a high content of microvoids as revealed by SAXS. Third, the end-product is a porous material consisting mainly of paraffinic chain crystals terminated by carboxyl groups. In this study we set out to examine: 1) the change of microhardness as a function of acid exposure in terms of the above structural scheme; 2) to follow the increase in hardness after recrystallization of the polymer residues from the melt. The hardening of treated PE after recrystallization was briefly discussed in an earlier publication [12].

\section{Experimental details}

The work has been carried out in three polyethylene materials previously characterized [9-11]. Values of molecular weight, degree of branching, crystallinity and lamellar thickness (as derived from long period) are collected in Table 1. Fuming nitric acid exposure of 
Table 1. Molecular weight $M_{w}$, branching concentration $\varepsilon$, crystallinity $\alpha$, lamellar crystal thickness $l$, and microhardness $\mathrm{MH}$ for three commercial PE grades before and after 20 hours fuming nitric acid treatment

\begin{tabular}{llcclccc}
\hline & sample & $M_{w} \cdot 10^{-3}$ & $\varepsilon(\%)$ & $\alpha$ & $l_{t=o}(\mathrm{~nm})$ & $M H_{t=o}\left(\mathrm{MNm}^{-2)}\right.$ & $M H_{t=20 h}\left(\mathrm{MNm}^{-2}\right)$ \\
\hline Hoechst PA-130 & A & 12 & 1.81 & 0.71 & 11.8 & 75 & 45 \\
BASF a Wachs & B & 9.5 & 3.49 & 0.67 & 7.4 & 38 & 26 \\
Epolene C-12 & C & 11 & 6.90 & 0.21 & 3.9 & 5 & - \\
\hline
\end{tabular}

the samples was performed at $60^{\circ} \mathrm{C}$ within closed tubes for periods of 5-180 h [10]. The treated samples were washed in distilled water rinsed with acetone, and dried in vacuum. Further details are reported elsewhere [9]. The Vickers-hardness was measured using a $100 \mu \mathrm{m}$ height indenter. A load of $0.25 \mathrm{~N}$ and a loading cycle of 0.1 minute were employed to minimize creep effects.

\section{Microhardness $(\mathrm{MH})$ of fuming nitric acid treat- ed polyethylene}

Microhardness of the untreated materials (see Table 1) is an increasing function of lamellae thickness and crystallinity. Such a dependence has recently been discussed in terms of the plastic deformation of crystals including a defective surface boundary (non-homogeneous microphase) $[13,14]$. Most conspicuous is the rapid decrease of $\mathrm{MH}$ as a function of fuming nitric acid exposure time (Table 2). This result contrasts with the hardening detected for $\mathrm{PE}$ treated with $\mathrm{H}_{2} \mathrm{SO}_{4}$ and $\mathrm{ClHSO}_{3}[3,4]$. While in the former case the reaction involves a substantial removal of uncrystallized material leaving a multitude of microvoids in the later cases the electron dense group attachment at the lamellar surface causes a weight increase, reinforcing the mechanical stability of the crystals [3]. Reliable $\mathrm{MH}$ values could not be obtained for exposure periods $t>$ $20 \mathrm{~h}$ due to the increasing brittleness of the degraded polymer. Figure 1 shows the $\mathrm{MH}$ decrease as a function of volume-percent of microvoids, $\Delta V$, for samples $\mathrm{A}$ and $\mathrm{B}$. Measurement of $\mathrm{MH}$ for sample $\mathrm{C}$

Table 2. Microhardness of samples A and B as a function of exposure time to fuming nitric acid

\begin{tabular}{rll}
\hline$t(h)$ & $\begin{array}{ll}\mathrm{MH}\left(\mathrm{MNm}^{-2}\right) \\
\mathrm{A}\end{array}$ & $\mathrm{B}$ \\
\hline 0 & 75 & 38 \\
5 & 55 & 26 \\
10 & 50 & 24 \\
15 & - & 20 \\
20 & 45 & 26 \\
\hline
\end{tabular}

was not feasible even for short treatment periods. The value of $\Delta V$ was estimated from the weight loss of material (mainly amorphous) [10] and the measured densities of the treated samples [15]. From the emerging small angle X-ray continuous scattering in the degraded samples microvoids in the range of 4-20 nm can be inferred [15]. A first rapid decrease of $~ 30 \%$ in $\mathrm{MH}$ for $\Delta V \sim 5 \%$ and a final leveling off of $\mathrm{MH}$ for $\Delta V$ $>10 \%$ is obtained for samples $A$ and $B$ in Figure 1. The acid exposure times are given in the figure. The final $\mathrm{MH}$ values are markedly lower than expected for the corresponding crystal hardness $H_{c}=M H / \alpha$ (arrows in Fig. 1). The large difference between the microhardness of the treated material and $H_{c}$ can be attributed to

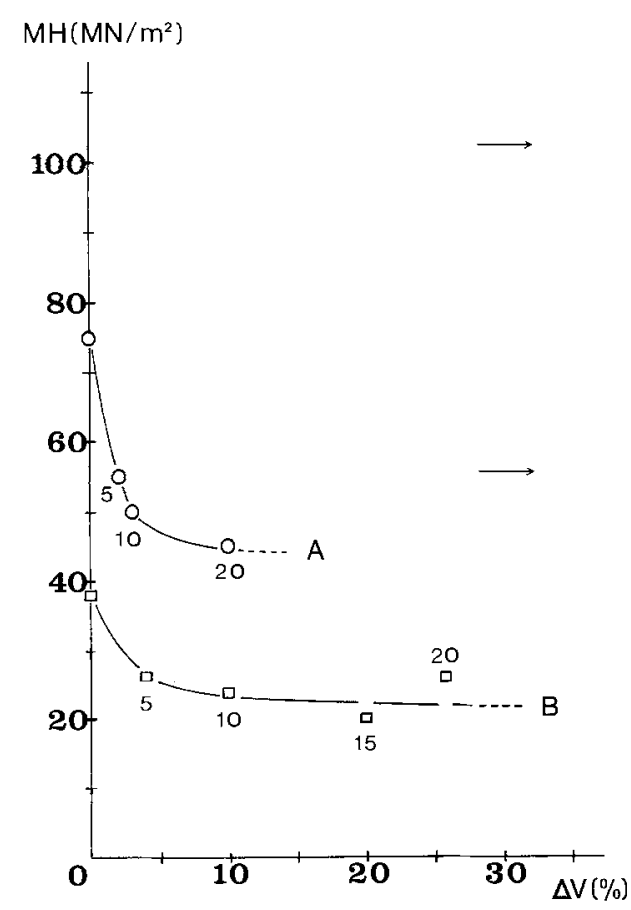

Fig. 1. Microhardness of polyethylene samples $A$ and $B$ as a function of microvoid percent $\Delta V$. Exposure times to fuming nitric acid, in hours, are given. The crystal hardness values $H_{c}=M H / \alpha$ for the untreated samples are indicated by arrows 


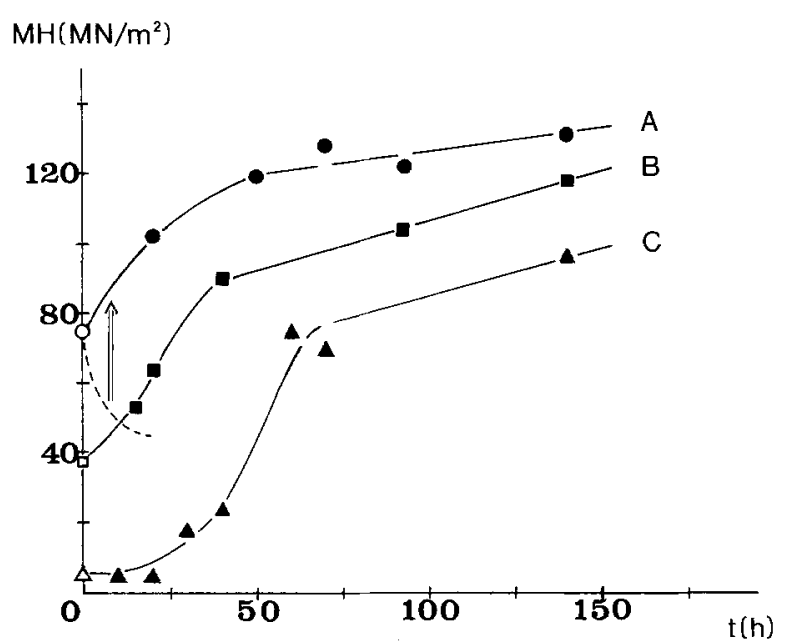

Fig. 2. Microhardness increase of fuming nitric acid treated PE samples after recrystallization from the melt as a function of treatment time. Open symbols: untreated samples. The initial microhardness decrease is only represented, for comparison, (dashed curve) for the treated sample $A$

the lack of an efficient packing of the hard lamellar crystals, now separated by a multitude of interlamellar microvoids. Up to $20 \mathrm{~h}$ of acid exposure the samples A and $B$ in Figure 1 still contain half the volume of uncrystallized regions contributing to irreversible modes, such as plastic flow, under the indenter. However, for longer treatment times the contribution of the vanishing amorphous layer, acting as a true interlamellar bridging component, becomes negligible and the macroscopic fragility of the material rapidly increases. Here indentation induced microcracking of the material leads to disruptive effects of the overall deformation pattern. For long acid exposure-times the powdery sample collapses under the indenter and an estimation of $\mathrm{MH}$ is not anymore feasible.

\section{Hardening behaviour after recrystallization}

To improve the overall surface mechanical properties of the degraded material the samples were recrystallized from the melt between glass plates. Figure 2 illustrates the initial hardness decrease after acid treatment (dashed curve) followed by the hardening obtained after recrystallization (arrow) for sample A. In this way the porous structure of the treated polymer is transformed into a hard material exhibiting a surface with a high reflectance amenable to yield distinct irre- versible deformation patterns. Figure 2 also shows the overall $\mathrm{MH}$ increase obtained for the three materials after recrystallization, as a function of prolonged exposure time. Two distinct hardening regions can be distinguished: A region yielding a rapid $\mathrm{MH}$ increase during the first $50 \mathrm{~h}$ of treatment. The hardening rate here is of about $1 \mathrm{MNm}^{-2} \mathrm{~h}^{-1}$, similar to that observed when exposing $\mathrm{PE}$ to $\mathrm{ClHSO}_{3}$ [3]. A second region for $t>$ $50 \mathrm{~h}$ shows a substantially lower MH increase. The following contributions to the overall $\mathrm{MH}$ increase are suggested:

Firstly, the removal of an increasing amount of disordered material (soft component) rising with exposure time. Before $t<50 \mathrm{~h}$ the recrystallized material continues to behave mechanically like a polymer.

Secondly, the concurrent fixation of oxydized groups-COOH, $-\mathrm{ONO}_{2},-\mathrm{NO}_{2}$ at the lamellae surface [9]. After recrystallization the dicarboxylic chain molecules with a given length distribution will tend efficiently to pack through association of the carboxyl groups at the crystal surface [7]. In these systems a segregation process leading to a complex multicomponent extended three-dimensional mixed lamellar structure has been proposed $[16,17]$. The packing of extended chain lamellae terminated predominantly by carboxylic groups will contribute to an additional hardening of the recrystallized material. A further support for the influence of the dicarboxylic groups at the crystal surface is provided by the decrease $(5-40 \%)$ of the rate of creep under the indenter measured after recrystallization. A similar effect has been reported for PE hardened with $\mathrm{ClHSO}_{3}$ [3].

Thirdly, the digestion of thinner and less perfect crystals only leaving the larger dicarboxylic chain segments to recrystallize [15].

The combination of these three contributions should give the hardening behaviour of Figure 2. The first contribution will predominate for $t<50 \mathrm{~h}$. The 2nd and third contributions will be present at shorter times but will prevail for $t>50 \mathrm{~h}$ i. e. the material behaves more like a long chain paraffin terminated by dicarboxylic groups. Contributions 2 and 3 are, additionally, in consonance with the increase of melting temperature of the treated samples [11].

\section{Comparison of experimental and calculated data}

Finally, it is helpful to examine the above reported data in the light of recent thermodynamic predictions [13]. Accordingly crystal hardness $H_{c}$ of materials 


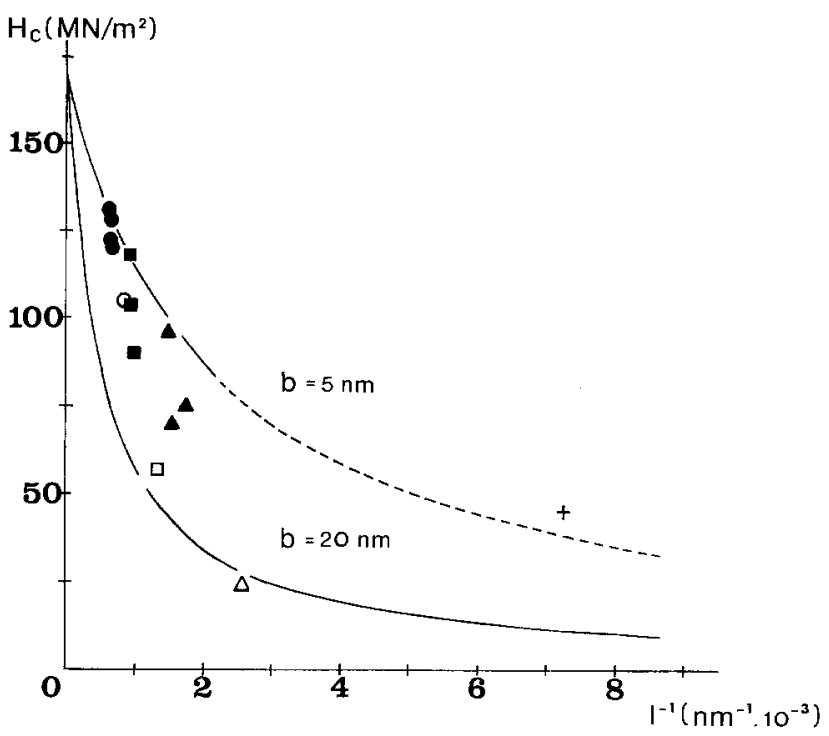

Fig. 3. Crystal hardness $H_{c}$ as a function of reciprocal crystal thickness $l$ according to equation 1 for $b=20 \mathrm{~nm}$ (melt crystallized PE and paraffins) [13] and $b 5=\mathrm{nm}$ (dicarboxylic acids). Open symbols: untreated samples. Full symbols: FNA treated samples for $t \geq$ 50 h. A $(O, \ominus), \mathrm{B}(\square, \square), \mathrm{C}(\Delta, \mathbf{A})$. Dodecanedioic acid $(+)$

consisting of lamellar structures with thickness, $l$, can be described through:

$$
H_{c}=\frac{H_{o}}{\left(1+b l^{-1}\right)}
$$

where $H_{o}=170 \mathrm{MNm}^{-2}$ is the hardness of an infinitely thick crystal and $b \simeq 20 \mathrm{~nm}$ under normal crystallization conditions. Figure 3 shows the $H_{c}$ predictions, according to Equation (1), for melt crystallized polyethylene and paraffinic crystals (lower curve) $(b \simeq 20$ $\mathrm{nm})$ and for dicarboxylic long-chain crystals assuming a value of ( $b \simeq 5 \mathrm{~nm}$ ) (upper curve). The hardness $H_{c}$ for the dodecanedioic acid is not far off from this curve. The data for the untreated samples (open symbols) lie well on the predicted curve, except for sample A which shows a somewhat higher value. This deviation is similar to that observed for other low molecular weight grades developing a high degree of crystallinity. The data for the degraded samples, after recrystallization, approach the curve with $b \simeq 5 \mathrm{~nm}$ for long treatment times $(t \sim 150 \mathrm{~h})$. Comparison of the data in terms of both predictions allows a way to separate between the influence of $l$ (abcissa) and the effect of carboxyl groups (ordinate) on the hardening of the material after acid attack and subsequent recrystallization. The results of Figure 3 suggest that the major contribution in the hardening after long treatment times is due to the attachment of electron dense groups, acting as defects in the longitudinal boundary layers of these extended carboxylic chain crystals. The hardening owing to the removal of thinner crystals during the nitric acid exposure seems to play a minor role. Nevertheless, the intermediate $H_{c}$ values between the lower and the upper curve in Figure 3 most probably involve structures of increasing perfection, better chain-end packing and lower crystal polydispersity. The independent influence of crystal shape and symmetry [18] on the posible additional hardness enhancement of dicarboxylic chain crystals will be the object of a further study.

\section{Conclusions}

In conclusion, the degradation mechanism of polyethylene exposed to fuming nitric acid can be adequately followed by the microhardness technique. The decrease in $\mathrm{MH}$ observed after nitric acid exposure is related to the volume increase of microvoids within the material. Recrystallization of the degraded polymer results in a substantially harder material yielding values in the vicinity of those obtained for polyethylene treated with chlorosulphonic and/or sulfuric acid. The hardening of recrystallized material is mainly related to the attachment of dicarboxylic groups between the boundary layers of the extended chain crystals.

\section{Acknowledgement}

This work was generously supported by CAICYT, Spain.

\section{References}

1. Baltá Calleja FJ (1985) Adv Polym Sci 66:117

2. Baltá Calleja FJ, Rueda DR, Garcia J, Wolf FP, Karl HV (1986)J Mater Sci 21:1139

3. Martinez Salazar J, Rueda DR, Cagiao ME, Lopez Cabarcos E, Baltá Calleja FJ (1983) Polym Bull 10:553

4. Baltá Calleja FJ, Fonseca C, Pereña JM, Fatou JG (1984) J Mater Sci Lett 3:509

5. Palmer RP, Cobbold AJ (1964) Makromol Chem 74:174

6. Keller A, Sawada S (1964) Makromol Chem 74:190

7. Illers KH (1968) Makromol Chem 118:88

8. Meinel G, Peterlin A (1967) J Polym Sci B 5:197

9. Rueda DR, Cagiao ME, Baltá Calleja FJ (1981) Makromol Chem 182:2705

10. Cagiao ME, Rueda DR, Baltá Calleja FJ (1980) Polym Bull 3:305

11. Cagiao ME, Rueda DR, Baltá Calleja FJ (1983) Coll \& Polym Sci 261:626

12. Baltá Calleja FJ (1976) Coll \& Polym Sci 254:258 
13. Baltá Calleja FJ, Kilian HG (1985) Coll \& Polym Sci 263:697

14. Baltá Calleja FJ, Kilian HG (1985) In: Kleintjens LA, Lemstra PJ (eds) Integration of Fundamental Polymer Sci and Technology, Elsevier, New York, p 517

15. Cagiao ME (1980) Ph D Thesis, Univ Compl Madrid

16. Asbach GI, Derexhage KH, Heidemann G, Glenz W, Kilian HG (1970) Makromol Chem 139:115

17. Kilian HG (1979) Makromol Chem Suppl 3:277

18. Ania F, Kilian HG, Baltá Calleja FJ (1986)JMater Sci Lett 5:1183
Received May 21, 1986; accepted June 9, 1986

Authors' address:

Prof. Dr. F. J. Baltá Calleja

Instituto de Estructura

de la Materia, CSIC

Serrano 119

28006 Madrid, España 